\title{
Sensitivity and uncertainty analysis of a simplified Kirschner-Panetta model for immunotherapy of tumor-immune interaction
}

\author{
Onyango Lawrence Omondi ${ }^{1,2^{*}}$, Chuncheng Wang ${ }^{1}$ and Xiaoping Xue
}

"Correspondence:

lawionyi@gmail.com

1 Department of Mathematics,

Harbin Institute of Technology,

Harbin, 150001, P.R. China

${ }^{2}$ Department of Mathematics,

Egerton University, P.O. Box 536

Egerton, 20115, Kenya

\section{Springer}

\begin{abstract}
In this study, we have simplified the Kirschner-Panetta model on the interaction of tumor cells and effector cells by considering linear growth term as opposed to logistic growth term used by Kirschner and Panetta. We have done a comprehensive mathematical analysis and established the existence of positive equilibrium. In addition, a fixed point bifurcation is investigated using the rate of spread of tumor as a varying parameter, suggesting that backward bifurcation can occur under reasonable choice of parameters. However, bistable dynamics is unlikely to happen in this case, which implies that the strategies that could reduce the rate of spread of tumor are the most influential to cancer treatment. Through mathematical deduction and numerical simulation, an elaborate uncertainty and sensitivity analysis of the rate of spread of tumor $R_{S}$ is performed. The distribution of $R_{s}$ is derived, and the sensitivity of the magnitude of $R_{s}$ to the uncertainty in estimating values of input parameters is assessed. The results indicate that the external source of effector cells and its death rate are influential in the rate of spread of tumor.
\end{abstract}

Keywords: tumor-immune system; backward bifurcation; uncertainty and sensitivity analysis

\section{Introduction}

Cancer is a term used to describe a disease in which abnormal cells divide without control and are able to invade other tissues. Cancer cells can spread to other parts of the body through blood and lymph systems. The main categories of cancer include carcinoma, sarcoma, leukemia, lymphoma, and myeloma [1-3]. Cancer is known as the leading cause of deaths in the world, and research has shown that new cases of cancer are on the rise. For instance, in 2008, there were estimated 12,667,500 new cases of cancer worldwide, with Eastern Asia having most of the cases $(3,720,000)$. It has also been observed that one eighth of deaths in the world is due to cancer. In fact, it causes more deaths than AIDS, tuberculosis, and malaria combined [4-7].

Every person has cancer cells in his body, but these may not show up in the standard clinical tests until they multiply to a few billions $[4,8]$. Clinically, cancer treatment has been a major challenge to medical practitioners, and no known cure has so far been found. Hence, when a doctor tells a patient that there are no more cancer cells in his body after

(c) 2015 Omondi et al. This article is distributed under the terms of the Creative Commons Attribution 4.0 International License (http://creativecommons.org/licenses/by/4.0/), which permits unrestricted use, distribution, and reproduction in any medium, provided you give appropriate credit to the original author(s) and the source, provide a link to the Creative Commons license, and indicate if changes were made. 
Figure 1 Depiction of loss of normal growth control, see [4].

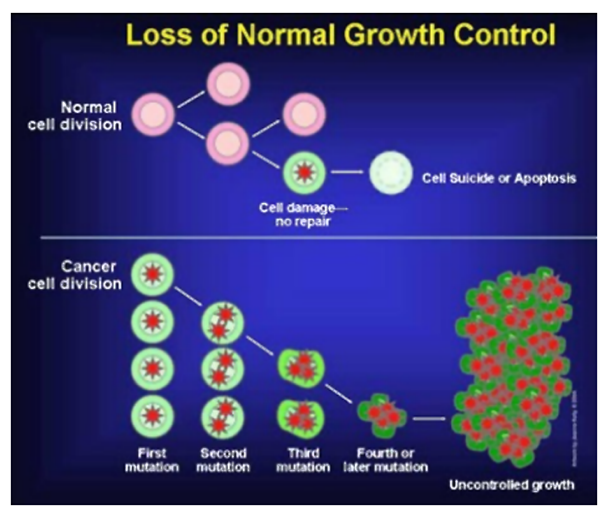

treatment, it basically means that the tests are unable to detect the cancer cells because they have gone below detectable levels $[4,9,10]$. All cancers begin in the cells, the body unit of life. To understand cancer, it is helpful to know what happens when a normal cell becomes a cancer cell. The body is made up of many types of cells, for example, blood cells (white and red), nerve cells, epithelial, and muscle cell amongst others. These cells grow and divide in a controlled way to produce more cells as they are needed to keep the body healthy. When cells become old and damaged, they die and are replaced with new ones. However, sometimes this orderly process goes wrong. The genetic material (DNA) of a cell can become damaged or changed, producing mutations that affect normal cell growth and division. When this happens, cells do not die when they should, and new cells form when the body does not need them. The extra cells form a mass of tissue called tumor as shown in Figure 1. Note, however, that not all tumors are cancerous, only a malignant tumor is cancerous [4].

\section{Motivation}

Kirschner and Panetta (KP) developed a cancer immunotherapy of tumor - immune systems model with logistic growth rate, and supported some of their findings through mathematical analysis and numerical simulations [11]. However, their formulations disregarded the rate of spread of tumor and the existence of a positive endemic equilibrium. Also, they did not identify the most influential parameters in their model. However, there is a need to address these two issues especially if the model is to be applied in a clinical setup. Rihan et al. tried to analyze the KP model (see [12]). They made certain assumptions, which reduced the KP model from a three-dimensional system of nonlinear ODEs to a two-dimensional one. However, explanation for the disappearance of the third variable is not provided. In addition, we observed that the type of non-dimensionalization pursued, which led to the elimination of the denominator variables and even transformation of some of the variables to parameters, fundamentally alters the KP model [12]. Moore studied the KP model and raised a concern that it inadequately represents the real situations [13].

The authors in [11] analyzed the KP model for tumors with logistic growth rate. However, testicular carcinomas, pediatric tumors, and some mesenchymal tumors are examples of rapidly proliferating cell populations, for which the tumor volume doubling time (TVDT) can be counted in days, and they exhibit a linear growth rate [14]. In this work, therefore, we have considered a later case by setting constant growth rate term, $r_{2}(T)$, in 
the KP model. We have mathematically analyzed the KP model more comprehensively and proved that, indeed, a positive endemic equilibrium exists. Conditions for the occurrence of bifurcation are set out, and the type of anticipated bifurcation is specified. In addition, we have performed numerical simulations to confirm and demonstrate the validity of various assumptions and conclusions made during the analysis. The paper is organized as follows. In Section 1, we have provided some background information on cancer infection; in Section 2, we have given a brief discussion on the previous works about the KP model and provided some explanations regarding the necessity to analyze the model with a linear growth rate; in Section 3, we have presented the KP model and mathematically analyzed it; Section 4 presents the numerical simulations and discussions of the results to ground the assumptions and conclusions. The work is concluded in Section 5.

\section{The model}

The KP model [11] is given by the system

$$
\begin{aligned}
& \frac{d E}{d \tau}=c T-\mu_{2} E+\frac{p_{1} E L}{g_{1}+L}+s_{1}, \\
& \frac{d T}{d \tau}=r_{2} T-\frac{a E T}{g_{2}+T}, \\
& \frac{d L}{d \tau}=\frac{p_{2} E T}{g_{3}+T}-\mu_{3} L+s_{2} .
\end{aligned}
$$

In the above system, and on the strength of the presentation by Kirschner and Panetta [11], in which $L(\tau)$ is the concentration of IL-2, which is the interleukin-2, the main cytokine responsible for lymphocyte activation, growth and differentiation; $E(\tau)$ represents the activated immune cells commonly called effector cells such as cytotoxic T-cells, microphages, and natural killer cells that are cytotoxic to tumor cells; microphages and natural killer cells that are cytotoxic to tumor cells; $T(\tau)$ is the tumor cells; with initial conditions $E(0)=E_{0}, T(0)=T_{0}, L(0)=L_{0}$. The parameter $c$ represents the antigenicity of tumor; $s_{1}$ is the treatment term that represents an external source of effector cells such as LAK and TIL cells; $s_{2}$ is the treatment term that represents external input of IL-2 into the system; $r_{2}$ is the linear growth rate; $1 / \mu_{2}$ is the average natural lifespan (in days) of effector cells; $\mu_{3}$ is the loss rate of IL-2; $a$ is the strength of immune response, while the rest of the parameters, namely $g_{1}, g_{2}$, and $g_{3}$, are substantive nonnegative thresholding parameters which are given in volumes. Non-dimensionalizing system (1) according to the following scaling regime (see [15])

$$
\begin{array}{ll}
x=\frac{E}{g_{2}}, & y=\frac{T}{g_{2}}, \quad z=\frac{L}{g_{1}}, \quad t=\tau t_{0}, \\
\hat{c}=\frac{c}{t_{0}}, & \hat{\mu}_{2}=\frac{\mu_{2}}{t_{0}}, \quad \hat{p}_{1}=\frac{p_{1}}{t_{0}}, \quad \hat{s}_{1}=\frac{s_{1}}{g_{2} t_{0}}, \\
\hat{r}_{2}=\frac{r_{2}}{t_{0}}, & \hat{a}=\frac{a}{t_{0}}, \quad \hat{p}_{2}=\frac{p_{2} g_{2}}{g_{1} t_{0}}, \\
\hat{g}_{3}=\frac{g_{3}}{g_{2}}, & \hat{\mu}_{3}=\frac{\mu_{3}}{t_{0}}, \quad \hat{s}_{2}=\frac{s_{2}}{t_{0} g_{1}},
\end{array}
$$

we obtain

$$
t_{0} \frac{g_{2} d x}{d t}=c g_{2} y-\mu_{2} g_{2} x+\frac{p_{1} g_{2} g_{1} x z}{g_{1}+g_{1} z}+s_{1}
$$




$$
\begin{aligned}
& \frac{d x}{d t}=\frac{c y}{t_{0}}-\frac{\mu_{2}}{t_{0}} x+\frac{\frac{p_{1}}{t_{0}}}{1+z} x z+\frac{s_{1}}{g_{2} t_{0}}, \\
& \frac{d x}{d t}=\hat{c} y-\hat{\mu_{2}} x+\frac{\hat{p_{1} x z}}{1-z}+\hat{s_{1}} .
\end{aligned}
$$

Applying the same procedure in the remaining two equations of system (1) and omitting the hats for easy notations, we consequently obtain the following system:

$$
\left\{\begin{array}{l}
\frac{d x}{d t}=c y-\mu_{2} x+\frac{p_{1} x z}{1+z}+s_{1} \\
\frac{d y}{d t}=r_{2} y-\frac{a x y}{1+y} \\
\frac{d z}{d t}=\frac{p_{2} x y}{g_{3}+y}-\mu_{3} z+s_{2}
\end{array}\right.
$$

It can be shown that (3) admits a tumor-free equilibrium

$$
P_{0}=\left(\frac{s_{1}\left(s_{2}+\mu_{3}\right)}{\mu_{2}\left(s_{2}+\mu_{3}\right)-p_{1} s_{2}}, 0, \frac{s_{2}}{\mu_{3}}\right)
$$

if $\mu_{2}\left(s_{2}+\mu_{3}\right)-p_{1} s_{2}>0$. The Jacobian matrix $J$ of the linearization of (3) at this equilibrium is given by

$$
J_{\left(x_{0}, y_{0}, z_{0}\right)}=\left[\begin{array}{ccc}
\frac{-\mu_{2}\left(s_{2}+\mu_{3}\right)+p_{1} s_{2}}{\left(s_{2}+\mu_{3}\right)} & c & \frac{p_{1} s_{1} \mu_{3}^{2}\left(s_{2}+\mu_{3}\right)}{\left(s_{2}+\mu_{3}\right)^{2}\left[\mu_{2}\left(s_{2}+\mu_{3}\right)-p_{1} s_{2}\right]} \\
0 & r_{2}-\frac{a s_{1}\left(s_{2}+\mu_{3}\right)}{\mu_{2}\left(s_{2}+\mu_{3}\right)-p_{1} s_{2}} & 0 \\
0 & \frac{p_{2} s_{1}\left(s_{2}+\mu_{3}\right)}{p_{3}^{2} \mu_{2}\left(s_{2}+\mu_{3}\right)-p_{1} s_{2}} & -\mu_{3}
\end{array}\right] .
$$

From (5) we obtain the following three real eigenvalues:

$$
\lambda_{1}=\frac{p_{1} s_{2}-\mu_{2}\left(s_{2}+\mu_{3}\right)}{s_{2}+\mu_{3}}, \quad \lambda_{2}=r_{2}-\frac{a s_{1}\left(s_{2}+\mu_{3}\right)}{\mu_{2}\left(s_{2}+\mu_{3}\right)-p_{1} s_{2}}, \quad \lambda_{3}=-\mu_{3}<0 .
$$

At each time step, a tumor cell either produces an offspring or dies. If each cell produces on average more than one new cell, then the basic reproduction number, $R_{s}$, which measures the rate of spread of tumor, is greater than one, $R_{s}>1$, and the tumor grows over time. If $R_{s}<1$, then each cell produces on average less than one new cell, and therapy can eradicate the tumor [16].

To calculate $R_{s}$, we use the next generation operator approach [17-20] and obtain

$$
R_{s}=\frac{r_{2} \mu_{2}\left(s_{2}+\mu_{3}\right)-r_{2} p_{1} s_{2}}{a s_{1}\left(s_{2}+\mu_{3}\right)} .
$$

To obtain the interior equilibrium, we equate each of the equations of system (3) to zero, from which we find that

$$
x=\frac{r_{2}}{a}(1+y), \quad z=\frac{p_{2} r_{2} y(1+y)}{a \mu_{3}\left(g_{3}+y\right)}+\frac{s_{2}}{\mu_{3}} .
$$

Substituting (8) into the steady state system from (3), we obtain

$$
A_{3} y^{3}+A_{2} y^{2}+A_{1} y+A_{0}=0
$$


where

$$
\begin{aligned}
A_{3}= & p_{2} r_{2}\left(p_{1} r_{2}+a c-\mu_{2} r_{2}\right), \\
A_{2}= & p_{2} r_{2}\left(p_{1} r_{2}+a s_{1}-\mu_{2} r_{2}\right)+\left(p_{2} r_{2}+a \mu_{3}+a s_{2}\right)\left(p_{1} r_{2}+a c-\mu_{2} r_{2}\right)-p_{1} r_{2} a \mu_{3}, \\
A_{1}= & \left(p_{2} r_{2}+a \mu_{3}+a s_{2}\right)\left(p_{1} r_{2}+a s_{1}-\mu_{2} r_{2}\right)-p_{1} r_{2} a \mu_{3} \\
& +a g_{3}\left(s_{2}+\mu_{3}\right)\left(p_{1} r_{2}+a c-\mu_{2} r_{2}\right)-p_{1} r_{2} a \mu_{3} g_{3}, \\
A_{0}= & a g_{3}\left(s_{2}+\mu_{3}\right)\left(p_{1} r_{2}+a s_{1}-\mu_{2} r_{2}\right)-p_{1} r_{2} a \mu_{3} g_{3} .
\end{aligned}
$$

The rate of spread of tumor $R_{s}$ and the behavior of the coefficients $A_{0}, A_{1}, A_{2}$, and $A_{3}$ of the cubic equation above lead us to the statement and proof of the following theorem.

Theorem 1 Assume that $\mu_{2}\left(s_{2}+\mu_{3}\right)-p_{1} s_{2}>0$. System (3) has a unique tumor-free equilibrium $P_{0}$, which is stable for $R_{s}<1$ and unstable for $R_{s}>1$. Furthermore, if $p_{2} r_{2}\left(p_{1} r_{2}+\right.$ $\left.a c-\mu_{2} r_{2}\right)>0\left(p_{2} r_{2}\left(p_{1} r_{2}+a c-\mu_{2} r_{2}\right)<0\right)$, then system (3) has a unique positive solution when $R_{s}>1\left(R_{s}<1\right)$ and the following condition is satisfied:

$$
\text { if } A_{2}<0(>0) \text {, then } A_{1}<0(>0) \text {. }
$$

Proof It is easy to see that $\lambda_{1}<0$, therefore, the existence of boundary equilibrium and its stability is implied directly from (4) and (6), and the existence of interior equilibrium can be shown by Descartes's rule of signs [21, 22]. In fact, if $R_{s}>1$, then $A_{0}<0$. Note that we assume $A_{3}>0$; therefore, there are four possible sign changes of the coefficients of (9), that is, $(+,+,+,-),(+,+,-,-),(+,-,-,-)$, and $(+,-,+,-)$, and the last possibility can be ruled out by (10). By Descartes' theorem, the number of positive roots of (9) is either equal to the sign changes between consecutive coefficients, or is less than them by an even number, which completes the proof.

Note that when $R_{s}<1$, the spread of tumor is very slow and this type of tumor is called benign tumor. When $R_{s}>1$, the tumor spreads very fast. This type of tumor is called malignant tumor and it requires immediate treatment. As a special case, if $c=s_{1}$ or $g_{3}=1$, then (10) holds, which implies the existence and uniqueness of the positive equilibrium when $A_{3}>0\left(A_{3}<0\right)$ and $R_{s}>1\left(R_{s}<1\right)$. Moreover, if $g_{3}=1$, then (9) becomes a quadratic equation

$$
B_{2} y^{2}+B_{1} y+B_{0}=0
$$

with

$$
\begin{aligned}
B_{2} & =p_{2} r_{2}\left(p_{1} r_{2}+a c-\mu_{2} r_{2}\right), \\
B_{0} & =a\left(s_{2}+\mu_{3}\right)\left(p_{1} r_{2}+a s_{1}-\mu_{2} r_{2}\right)-p_{1} r_{2} a \mu_{3} \\
& =a^{2} s_{1}\left(s_{2}+\mu_{3}\right)\left(1-R_{0}\right), \\
B_{1} & =p_{2} r_{2}\left(p_{1} r_{2}+a s_{1}-\mu_{2} r_{2}\right)+a\left(s_{2}+\mu_{3}\right)\left(p_{1} r_{2}+a c-\mu_{2} r_{2}\right)-p_{1} r_{2} a \mu_{3} \\
& =B_{2}+B_{0}+a\left(s_{1}-c\right)\left(p_{2} r_{2}-a\left(s_{2}+\mu_{3}\right)\right),
\end{aligned}
$$

from which we can arrive at the following conclusion. 
Corollary 2 Assume that $\mu_{2}\left(s_{2}+\mu_{3}\right)-p_{1} s_{2}>0$ and $g_{3}=1$. When $B_{2}>0$, system (3) has a unique positive equilibrium if $R_{s}>1$, and has two positive equilibria if $R_{s}<1, B_{1}<0$, and $\Delta:=B_{1}^{2}-4 B_{0} B_{2}>0$. When $B_{2}<0$, system (3) has a unique positive equilibrium if $R_{s}<1$ and has two positive equilibria if $R_{s}>1, B_{1}>0$, and $\Delta>0$.

From Corollary 2, we expect that the backward bifurcation [23, 24] might occur, since one can prove that $B_{1}>0$ when $B_{2}>0$ and $0<R_{s} \ll 1$. In fact, for $0<R_{s} \ll 1$, we know that

$$
\begin{aligned}
B_{1} & =B_{2}+B_{0}+a\left(s_{1}-c\right)\left(p_{2} r_{2}-a\left(s_{2}+\mu_{3}\right)\right) \\
& \approx p_{2} r_{2}\left(p_{1} r_{2}+a c-\mu_{2} r_{2}\right)+a^{2} s_{1}\left(s_{2}+\mu_{3}\right)+a\left(s_{1}-c\right)\left(p_{2} r_{2}-a\left(s_{2}+\mu_{3}\right)\right) \\
& =p_{2} r_{2}\left(p_{1} r_{2}+a s_{1}-\mu_{2} r_{2}\right)+a^{2} c\left(s_{2}+\mu_{3}\right)>0 .
\end{aligned}
$$

Therefore, there is no positive equilibrium for system (3), which implies that the backward bifurcation occurs for (3) if $R_{s}$ is regarded as a variable parameter. However, the backward bifurcation does not exist for $s_{1}=c$ since $B_{1}=B_{0}+B_{2}$ in this case, and hence $B_{1}>0\left(B_{1}<\right.$ $0)$ if $B_{2}>0$ and $R_{s}<1\left(B_{2}<0\right.$ and $\left.R_{s}>1\right)$. Therefore, when $B_{2}>0\left(B_{2}<0\right)$, system (3) undergoes a supercritical (subcritical) fixed point bifurcation at $R_{s}=1$.

However, we observe from formulation (7) of the rate of spread of tumor $R_{s}$ that the dynamics of model (3) without the treatments $s_{1}$ and $s_{2}$ leads to a blow-up since $R_{s}=+\infty$. This implies that the treatments are very critical in controlling the spread of malignant cancer.

\section{Numerical simulations}

A sample set of reasonable parameter values for (1) can be found in [11]. After scaling those parameters according to (2), we get the set of parameter values for model (3) as in Table 1. Recall that $s_{1}$ is the treatment term that represents an external source of effector cell. We will use $s_{1}$ as a varying parameter to examine the effect of treatment on the number of tumor cells. It follows from (7) that $R_{s}$ is a decreasing function of $s_{1}$. With the values of parameters except $s_{1}$ given in Table 1 , one can obtain that $R_{s}>1$ when $s_{1}<0.0350$, $0.6384 \approx R^{*}<R_{s}<1$ when $0.0540>s_{1}>0.0350$, and $R_{s}<R^{*}$ when $s_{1}>0.0540$. Hence, freeing the parameter $s_{1}$ gives a bifurcation diagram (see Figure 2) with two positive equilibrium points, denoted by $\left(x_{1}, y_{1}, z_{1}\right)$ and $\left(x_{2}, y_{2}, z_{2}\right)$, for $0.0549>s_{1}>0.0350$ (or $R^{*}<R_{s}<$ 1), unique equilibrium for $s_{1}<0.0350$ (or $R_{s}>1$ ), and no equilibrium for $s_{1}>0.0549$ (or $R_{s}<R^{*}$ ). However, the positive equilibria, whenever they exist, are unstable since there exists at least one positive eigenvalue for a Jacobian matrix at each equilibrium, see Figure 3. Therefore, (3) does not have the bistable dynamics even though a backward bifurcation occurs. Set $s_{1}=0.04$. We use numerical simulations to examine the dynamics of (3), see Figure 2. It is observed that the solution will tend to the boundary equilibrium for $R_{s}<1$ and a blow-up solution exists for $R_{s}>1$, see Figure 4 .

Uncertainty and sensitivity analysis is conducted to explore the behavior of the model, because the model is structurally complex and the values of many input parameters are

Table 1 Parameter values used for model in (3)

\begin{tabular}{llllllllll}
\hline Parameter & $\boldsymbol{c}$ & $\boldsymbol{\mu}_{\mathbf{2}}$ & $\boldsymbol{p}_{\mathbf{1}}$ & $\boldsymbol{r}_{\mathbf{2}}$ & $\boldsymbol{a}$ & $\boldsymbol{p}_{\mathbf{2}}$ & $\boldsymbol{g}_{\mathbf{3}}$ & $\boldsymbol{\mu}_{\mathbf{3}}$ & $\boldsymbol{s}_{\mathbf{2}}$ \\
\hline Value & 0.025 & 0.16 & 0.69 & 1 & 4.5 & 0.2 & 1 & 7 & 0.05 \\
\hline
\end{tabular}


Figure 2 Bifurcation diagram of (3) with parameters given in Table 1. Here, the horizontal axis is the basic reproduction number, and the vertical axis is the number of tumor cells.

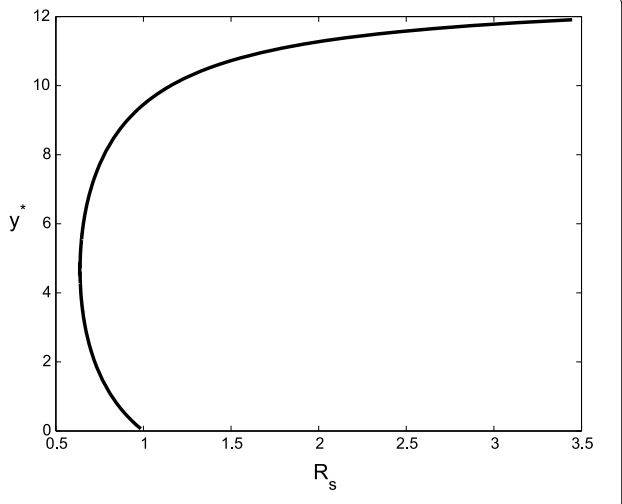

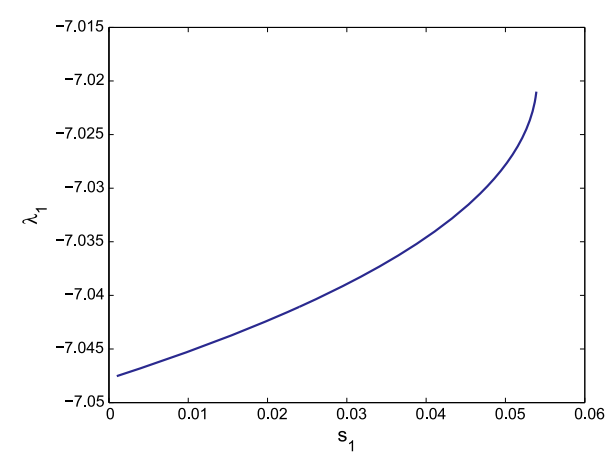
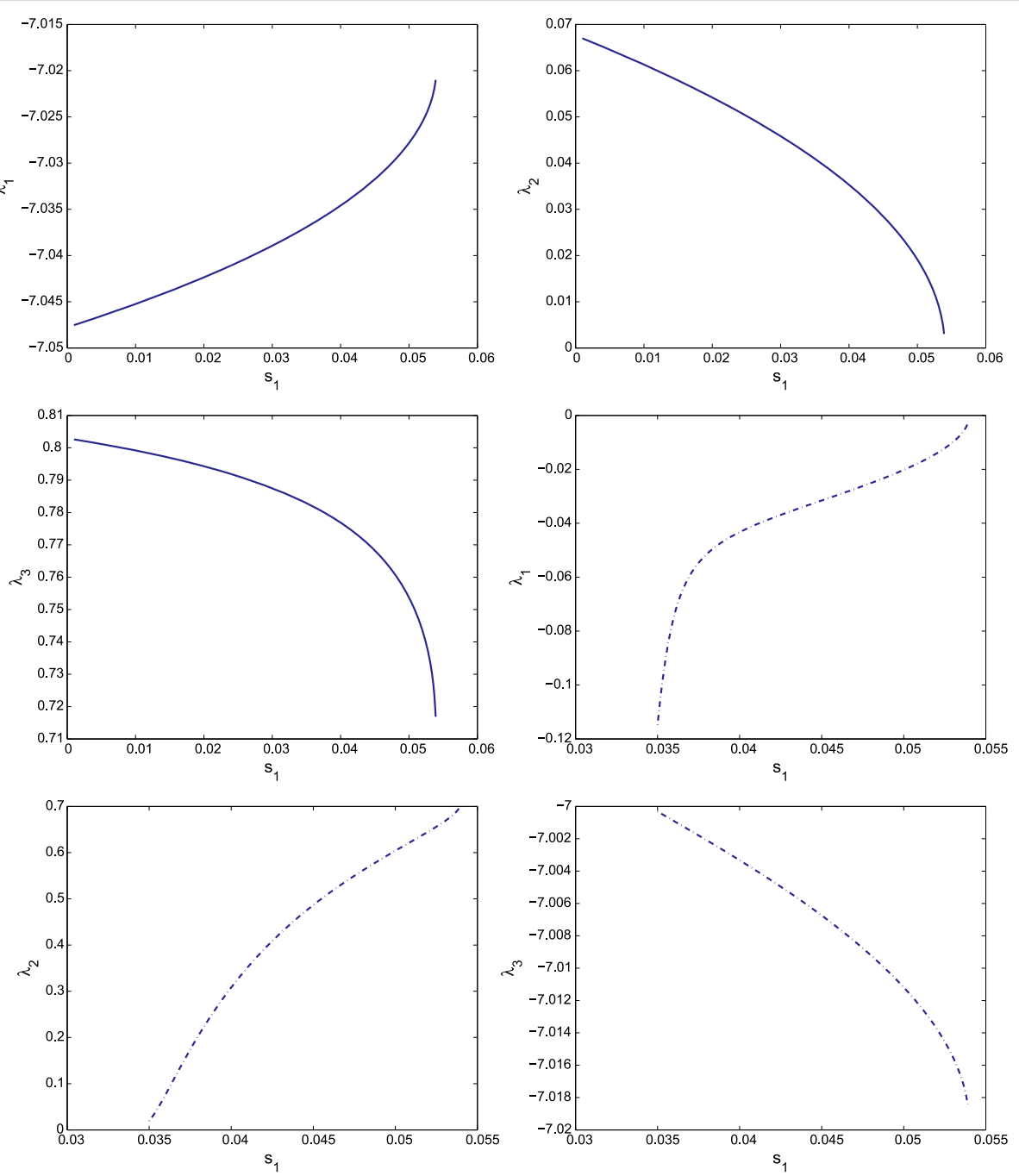

Figure 3 The eigenvalue of Jacobian matrix at the positive equilibrium varies as $s_{1}$ increases. The three solid lines represent the eigenvalues at the large equilibrium $\left(x_{1}, y_{1}, z_{1}\right)$, and the other three dash-dot lines represent the eigenvalues at the small equilibrium $\left(x_{2}, y_{2}, z_{2}\right)$. 

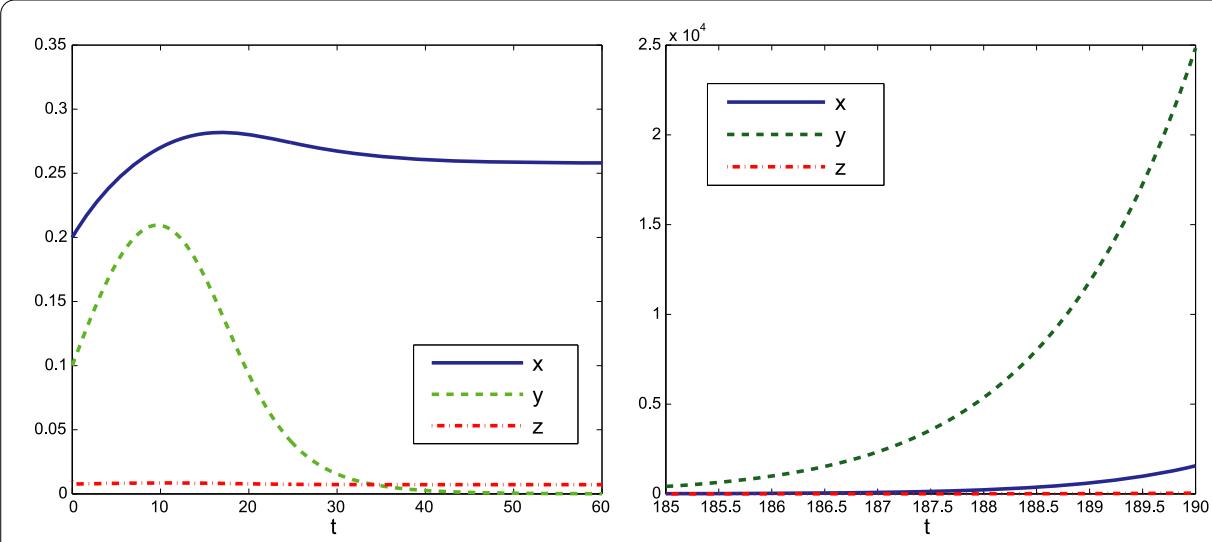

Figure 4 Numerical simulations of (3) with parameters given in Table 1 for various initial values. (Left) The solution tends to boundary equilibrium as $t$ goes to infinity for $R_{s}<1$; (Right) A blow up solution of model (3) for $R_{s}>1$.

estimated with a high degree of uncertainty. Uncertainty analysis can be used to assess the variability of the outcome variable ( $R_{s}$ for this model) that is due to the uncertainty of parameters in the model, and sensitivity analysis is an extension of uncertainty analysis that can identify which parameters are important to outcome variable. For uncertainty and sensitivity analysis, probability density functions (PDF) are assigned, in advance, to some parameters in the expression in $R_{s}$, and each specified PDF describes the range of possible values and the probability of occurrence of any specific value. Selection of triangular distribution is recommended when the most likely value and range of parameters are estimable. A uniform distribution is usually selected for the parameters when only a range for the parameter is estimable. Latin hypercube sampling is then used to sample these parameters based on their probability density functions. For system (3), seven parameters are selected and assigned to proper probability density functions (an asymmetric triangular probability distribution is selected for five of these parameters, and a uniform distribution is chosen for the other two parameters), and then 1,000 values for each parameter are sampled according to its PDF, see Figure 5. After the sampling, they are randomly permuted to form 1,000 input parameter vectors, which are used to explore the effect of uncertainty in estimating the values of the input variables on the prediction of the outcome variable, $R_{s}$, by (7). The distribution of all $R_{s}$ estimates is shown in Figure 6 . The descriptive statistics for the distribution is given in Table 2. The minimum of $R_{s}$ is 0.2408 , while its maximum is 3.9802 , which means that $R_{s}$ can be reduced below 1 for some specific choice of parameters. This implies that the tumor cells in the body can be eliminated with proper treatment strategy. Moreover, it can observed from Figure 6 that the percentage for $R_{s}$ less than 1 is higher than that for $R_{s}$ greater than 1 , which indicates that the factors $s_{1}$ and $s_{2}$ have positive effect on tumor treatment.

The partial rank correlation coefficient (PRCC), measuring the statistical influence (specifically the monotonicity) of parameters on $R_{s}$, is calculated between each of input parameters and the values of $R_{s}$. These PRCCs are used to identify the key input parameters that contributed to the prediction imprecision. The sign of PRCC identifies the specific qualitative relationship between the input and output variable, and the absolute value of PRCC indicates the degree of monotonicity between a specific input variable and the 

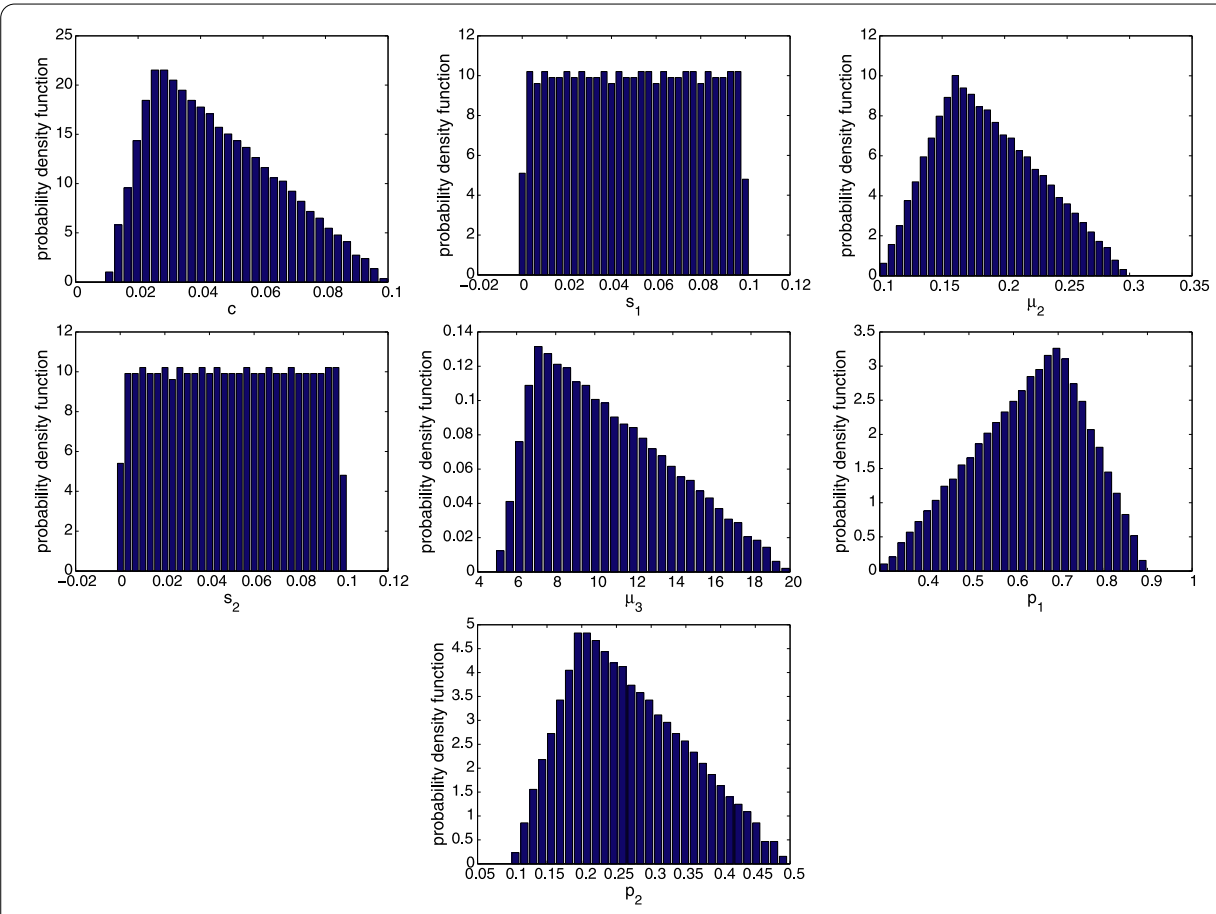

Figure 5 Histogram of the values obtained from Latin hypercube sampling for the seven input parameters.

Figure 6 Histogram of estimated $R_{s}$ values from Latin hypercube sampling.

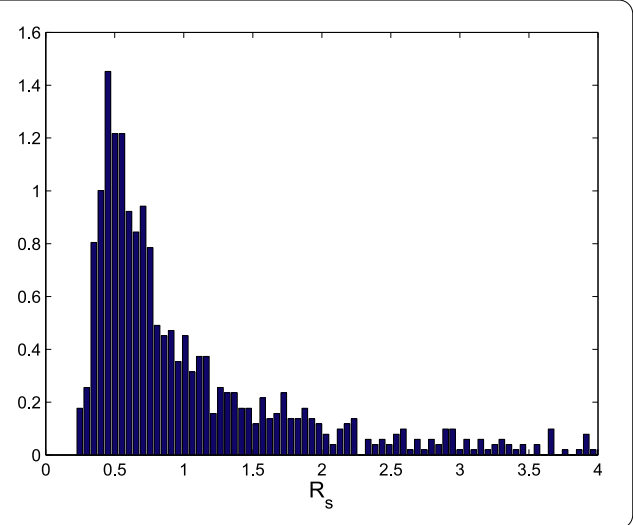

outcome variable. The PRCCs results are presented in Table 3, from which we can conclude that the first three parameters that contribute most to $R_{s}$ are $s_{1}, \mu_{2}$, and $s_{2}$. The uncertainties in estimating the values of these three parameters $\left(s_{1}, \mu_{2}\right.$, and $\left.s_{2}\right)$ are most influential in determining the prediction of $R_{s}(|\mathrm{PRCC}|>0.5)$ and are statistically significant $(p$-value $<0.05)$. The positive value of the PRCC implies that when the value of the input variable increases, the value of $R_{s}$ will also increase. Therefore, the increases in $s_{1}$ and $s_{2}$ correspond to a decrease in $R_{s}$, while an increase in $\mu_{2}$ corresponds to an increase in $R_{s}$. Note that $s_{1}$ is the external source of effector cells, $\mu_{2}$ is the loss rate of effector cells and $s_{2}$ is the treatment term that represents an external input of IL-2. This shows that the treatment strategy of increasing the external source of effector cells and IL-2 is the most effective way of tumor treatment. However, comparing with IL-2, the maintenance of a 
Table 2 Descriptive statistics from the uncertainty analysis

\begin{tabular}{lllll}
\hline & Minimum & Maximum & Mean & Variance \\
\hline$R_{5}$ & 0.2408 & 3.9802 & 0.8913 & 0.2150 \\
\hline
\end{tabular}

Table 3 Partial rank correlation coefficients (PRCCs) for the basic reproduction number and each input parameter variable

\begin{tabular}{llllllll}
\hline Parameter & $\boldsymbol{c}$ & $\boldsymbol{s}_{\mathbf{1}}$ & $\boldsymbol{\mu}_{\mathbf{2}}$ & \multicolumn{1}{c}{$\boldsymbol{s}_{\mathbf{2}}$} & $\boldsymbol{\mu}_{\mathbf{3}}$ & \multicolumn{1}{c}{$\boldsymbol{p}_{\mathbf{1}}$} & \multicolumn{1}{c}{$\boldsymbol{p}_{\mathbf{2}}$} \\
\hline PRCCs & 0.048 & -0.982 & 0.872 & -0.127 & 0.091 & -0.038 & -0.072 \\
$p$-value & 0.127 & 0 & 0 & $5.5 \times 10^{-5}$ & 0.004 & 0.229 & 0.022 \\
\hline
\end{tabular}

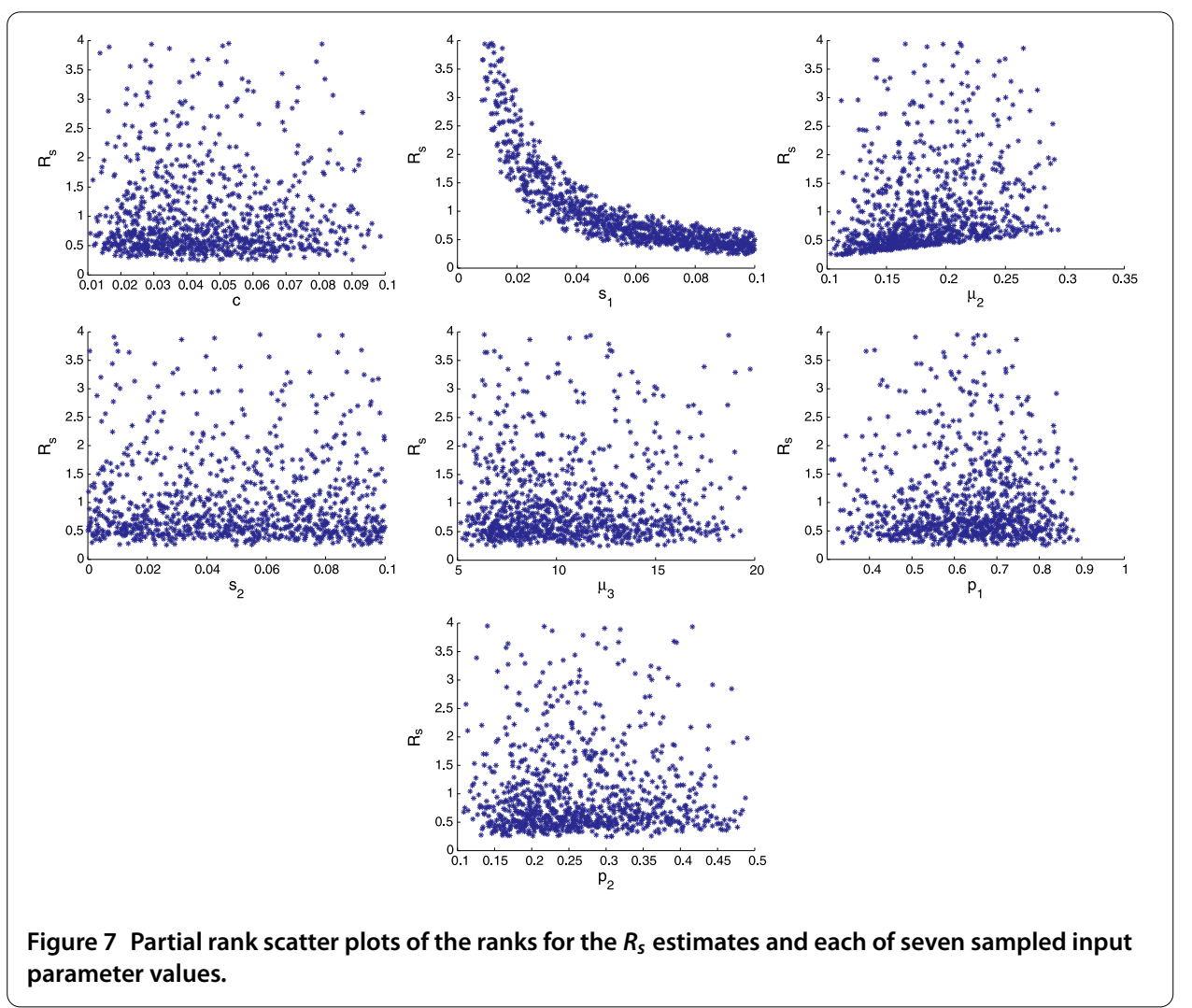

certain concentration of effector cells in the body is much more important since the absolute values of PRCCs of $s_{1}$ and $\mu_{2}$ are greater than 0.5. Partial rank scatter-plots for $R_{s}$ and each of the seven input variables are also generated, see Figure 7 . The monotonicity of $R_{s}$ with respect to $s_{1}$ and $\mu_{2}$, that can be observed in Figure 7, is clearly satisfied. These scatter-plots demonstrate how the rank transformation turns these monotonic relations into linear relations.

\section{Conclusion}

In this study, a mathematical analysis of the tumor-immune interaction model proposed in [11] has been simplified and analyzed. Proof of the existence of positive equilibrium has been established. Additionally, the rate of spread of tumor $R_{s}$ was derived, and explanations regarding the behavior of $R_{s}$ were provided. The fixed point bifurcation was investigated using $R_{s}$ as free parameters. The results reveal that backward bifurcation could 
occur for a reasonable set of parameter values. However, the numerical results indicate that the equilibria at the bifurcated branch are all unstable, meaning that the bistable dynamics will never happen for the model. Unlike most epidemic models in the literature, the numerical simulations in this work show that when $R_{s}>1$ the solution tends to infinity as time increases. Therefore, the control strategies (for instance, at least a certain amount of external effector $s_{1}$ ) that will reduce $R_{s}$ to within 1 are of great importance for the possible tumor cell elimination.

Due to the difficulty of measure, or collection, of empirical data on some parameters in the model, we also conducted uncertainty analysis and sensitivity analysis based on Latin hypercube sampling in estimating $R_{s}$. The distribution for $R_{s}$ gives a wide range of estimates due to uncertainty in estimating values of seven selected parameters. Even though the upper bound of $R_{s}$ could run up to 4 , the $95 \%$ confidence interval of the distribution of $R_{S}$ is $[0.2512,2.9341]$. Hence, tumor cells are likely to be eliminated under proper treatment strategies, based on the interpretation of the model, because this interval contains the number 1 . The results of sensitivity analysis imply that an increase in external effector would have lead to less severe growth of tumor size, because a high concentrate effector cells would have resulted in fewer number of tumor cells. A decrease in the per capita natural death rate of effector cells would also have led to fewer number of tumor cells. These results are obvious from the expression of (7), and our sensitivity analysis extends these conclusions quantitatively.

Competing interests

The authors declare that they have no competing interests.

Authors' contributions

OLO performed the mathematical analysis of the new model and also wrote and typeset the manuscript. CW identified the problem and conducted the numerical simulations. He also wrote the manuscript. XX proofread the final document. All authors read and approved the final manuscript.

\section{Acknowledgements}

The authors of this work are grateful to the journal editors and the anonymous reviewers for their comments and recommendations, which have greatly improved our manuscript and made it more suitable for readers of the journal. Additionally, we would like to appreciate B. Maiseli and E. Ogada for their contributions in editing the manuscript.

Received: 11 February 2015 Accepted: 18 June 2015 Published online: 11 July 2015

\section{References}

1. Varricchio, CG: A Cancer Source Book for Nurses. Jones \& Bartlett, Boston (2004)

2. Anand, P, Kunnumakara, AB, Sundaram, C, Harikumar, KB, Tharakan, ST, Lai, OS, Sung, B, Aggarwal, BB: Cancer is a preventable disease that requires major lifestyle changes. Pharm. Res. 25(9), 2097-2116 (2008)

3. Liu, Z, Romero-Canelón, I, Qamar, B, Hearn, JM, Habtemariam, A, Barry, NP, Pizarro, AM, Clarkson, GJ, Sadler, PJ: The potent oxidant anticancer activity of organoiridium catalysts. Angew. Chem. 126(15), 4022-4027 (2014)

4. Garcia, M, Jemal, A, Ward, E, Center, M, Hao, Y, Siegel, R, Thun, M: Global Cancer Facts \& Figures 2007, vol. 1. American Cancer Society, Atlanta (2007)

5. Jemal, A, Siegel, R, Xu, J, Ward, E: Cancer statistics, 2010. CA Cancer J. Clin. 60, 277-300 (2010)

6. Siegel, R, Ma, J, Zou, Z, Jemal, A: Cancer statistics, 2014. CA Cancer J. Clin. 64, 9-29 (2014)

7. Jemal, A, Ward, E, Thun, M: Declining death rates reflect progress against cancer PLoS ONE 5(3), 9584 (2010)

8. Ma, J, Siegel, R, Jemal, A: Pancreatic cancer death rates by race among US men and women, 1970-2009. J. Natl. Cancer Inst. 105(22), 1694-1700 (2013)

9. Hamilton, SR, Aaltonen, LA (eds.): World Health Organization Classification of Tumours: Pathology and Genetics of Tumours of the Digestive Syste. IARC press, Lyon (2000)

10. Ramaswamy, S, Tamayo, P, Rifkin, R, Mukherjee, S, Yeang, CH, Angelo, M, Ladd, C, Reich, M, Latulippe, E, Mesirov, JP, Poggio, T, Gerald, W, Loda, M, Lander, ES, Golub, TR: Multiclass cancer diagnosis using tumor gene expression signatures. Proc. Natl. Acad. Sci. USA 98(26), 15149-15154 (2001)

11. Kirschner, D, Panetta, JC: Modeling immunotherapy of the tumor-immune interaction. J. Math. Biol. 37(3), 235-252 (1998)

12. Rihan, FA, Safan, M, Abdeen, MA, Abdel-Rahman, DH: Mathematical modeling of tumor cell growth and immune system interactions. Int. J. Mod. Phys. Conf. Ser. 9, 95-111 (2012)

13. Moore, J: An ODE Model of Biochemotherapy Treatment for Cancer. PhD thesis, Department of Mathematics, Harvey Mudd College (2007) 
14. Friberg, S, Mattson, S: On the growth rates of human malignant tumors: implications for medical decision making. J. Surg. Oncol. 65(4), 284-297 (1997)

15. de Pillis, L, Radunskaya, A: Qualitative Analysis of Tumor-Immune ODE System (2002)

16. Michor, F, Nowak, MA, Iwasa, Y: Evolution of resistance to cancer therapy. Curr. Pharm. Des. 12(3), 261-271 (2006)

17. Diekmann, O, Heesterbeek, J, Metz, JA: On the definition and the computation of the basic reproduction ratio $R_{0}$ in models for infectious diseases in heterogeneous populations. J. Math. Biol. 28(4), 365-382 (1990)

18. Luchsinger, C: Einführung in die Mathematische Epidemiologie. Swiss Tropical and Public Health Institute (2011)

19. Keeling, MJ, Rohani, P: Modeling Infectious Diseases in Humans and Animals. Princeton University Press, Princeton (2008)

20. Anderson, RM, May, RM: Infectious Diseases of Humans, vol. 1. Oxford University Press, Oxford (1991)

21. Eigenwillig, A: Real Root Isolation for Exact and Approximate Polynomials Using Descartes' Rule of Signs. Dissertation (2008)

22. Stroud, KA, Booth, DJ: Advanced Engineering Mathematics. Palgrave Macmillan, Basingstoke (2003)

23. Wiggins, S: Introduction to Applied Nonlinear Dynamical Systems and Chaos, vol. 2. Springer, Berlin (2003)

24. Hirsch, MW, Smale, S, Devaney, RL: Differential Equations, Dynamical Systems and an Introduction to Chaos. Pure and Applied Mathematics, vol. 60. Elsevier, Amsterdam (2004)

\section{Submit your manuscript to a SpringerOpen ${ }^{\circ}$ journal and benefit from:}

- Convenient online submission

- Rigorous peer review

- Immediate publication on acceptance

- Open access: articles freely available online

- High visibility within the field

Retaining the copyright to your article 